\title{
'|||||||||||||||||||||||||||||||||||||||||||||||||||||||||||||||||||||.
}

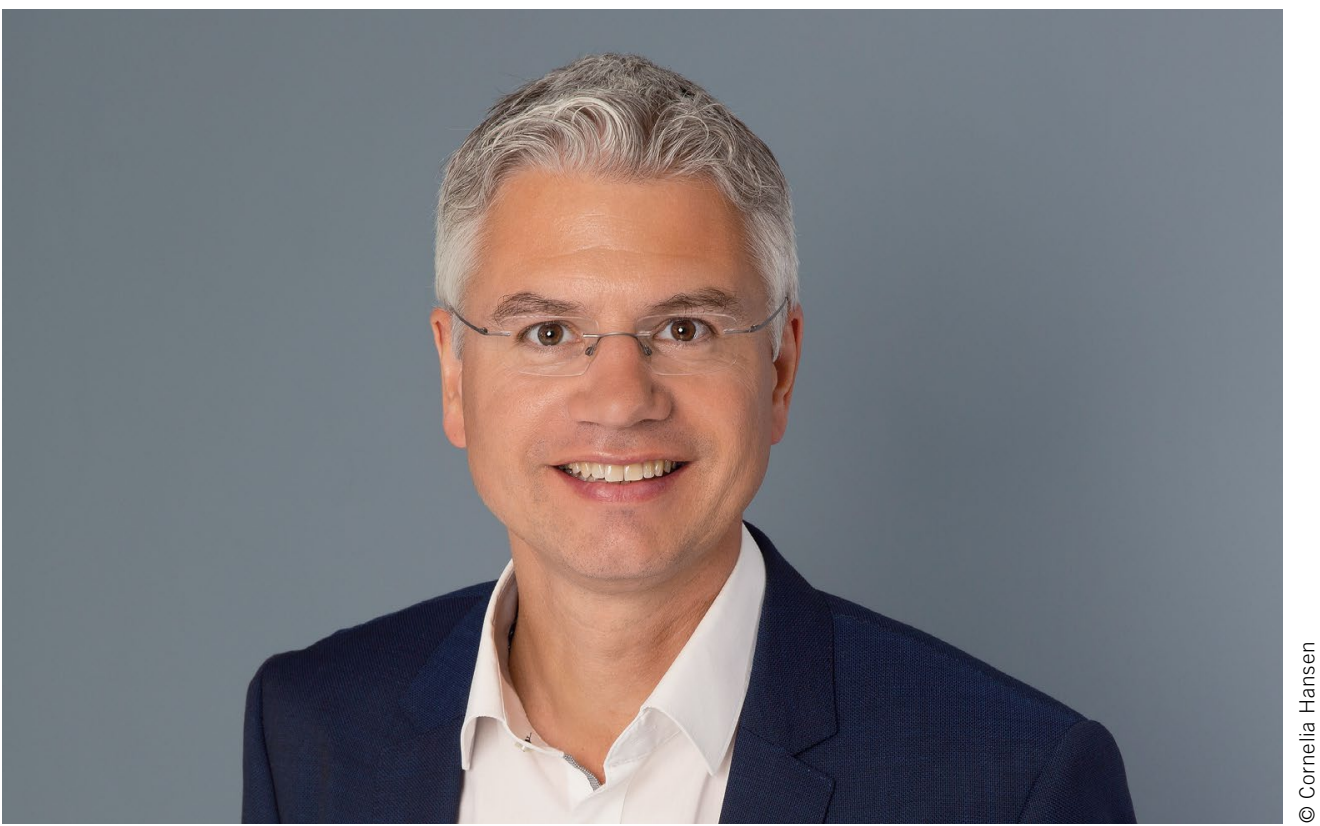

Prof. Dr.-Ing. Hanno Ihme-Schramm Department Fahrzeugtechnik und Flugzeugbau an der Hochschule für Angewandte Wissenschaften Hamburg

\section{Qualitätssicherung für Messdaten und Simulationsumgebungen}

Die virtuelle Entwicklungsumgebung in der Antriebsentwicklung ist in den vergangenen Jahren aus verschiedenen Gründen stärker in den Fokus gerückt. Sie bietet unter anderem die Möglichkeit, das Systemverständnis komplexer Antriebssysteme frühzeitig im Entwicklungsprozess zu erarbeiten und bei der bestehenden Vielfalt der Antriebsarten und -varianten Zeit und Kosten einzusparen.

Die aufwendige Erstellung der dazu benötigten Modelle und Simulationsumgebungen ist aber nicht immer zielführend. Das liegt häufig an der fehlenden Genauigkeit der virtuellen Entwicklungsumgebung zum realen Antrieb. Was sind die Gründe dafür? Einerseits kann die Spezifikation der Modellund Simulationsanforderungen unvollständig festgelegt oder umgesetzt sein, andererseits werden Modelle oft in späteren Anwendungsbereichen eingesetzt, die bei deren Erstellung noch nicht bekannt waren. Häufig stehen zur Validierung nur wenige Messdaten vom Prüfstand zur Verfügung, die darüber hinaus auch nicht immer zum Anwendungsgebiet passen. Wie genau sind diese Messdaten? Ist die Simulation oder sind die Daten fehlerhaft? Warum gibt es keine Qualitätsdokumentation für Messdaten und Simulationsumgebungen?

Um diese Fragen und Herausforderungen zu lösen, ist es aus meiner Sicht zwingend erforderlich, eine standardisierte und funktionierende Qualitätssicherung mit den Prozessschritten Messdatenplausibilität, Simulationsverifikation und -validierung zur Erstellung von Modellen und Simulationsumgebungen zu integrieren. Diese ist geprägt durch eine konsequente Auftragsklärung, Eigenverantwortung, standardisierte Dokumentation zu Anwendungsbereich und Qualität der Messdaten sowie Simulationsumgebungen und die stringente Kommunikation zwischen den beteiligten Personen. Nur so kann eine hohe Simulationsgenauigkeit und damit eine hohe Akzeptanz bei den Anwendern erreicht werden. Natürlich sind für den Aufbau der Qualitätssicherung für Messdaten und Simulationsumgebungen sowie deren Einsatz Personal, Zeit, Disziplin und Geld erforderlich. Letztendlich verkürzt sich so aber die Entwicklungszeit, lassen sich Kosten senken und die Qualität der Ergebnisse sichern.

Vonseiten der Hochschulen und Universitäten benötigen wir eine abgestimmte und ausgewogene Lehre für diese neue, hybride Entwicklungsumgebung. Die Tätigkeiten des Versuchsingenieurs und des Simulationsentwicklers sowie deren Zusammenarbeit im Entwicklungsprozess müssen im Studium gleichermaßen abgebildet werden. Dabei sollten die Studierenden Erfahrungen am Prüfstand sammeln und die Messdaten kritisch auf ihre Genauigkeit hin untersuchen. In den virtuellen Fächern lernen sie den Umgang mit den Themen Systemanforderung, Systemarchitektur, Modellbildung und die Erstellung von Simulationsumgebungen, die anschließend verifiziert und validiert werden. Dazu verwenden sie ihre selbst vermessenen Daten, die sie vorher plausibilisiert haben. 